\title{
Alternative functional in vitro models of human intestinal epithelia
}

\author{
Amanda L. Kauffman ${ }^{1}$, Alexandra V. Gyurdieva ${ }^{1}$, John R. Mabus ${ }^{1}$, Chrissa Ferguson ${ }^{2}, Z^{2 h e n g y i n}$ Yan $^{2}$ \\ and Pamela J. Hornby ${ }^{1 *}$
}

1 Biologics Research, Biotechnology Center of Excellence, Janssen Pharmaceutical Companies of Johnson \& Johnson, Spring House, PA, USA

2 Drug Discovery, Analytical Science, Janssen Pharmaceutical Companies of Johnson \& Johnson, Spring House, PA, USA

\section{Edited by:}

Angelo A. Izzo, University of Naples

Federico ii, Italy

Reviewed by:

Martin Diener, University Giessen,

Germany

Gabriella Aviello, Trinity College

Dublin, Ireland

*Correspondence:

Pamela J. Hornby, Biologics

Research, Biotechnology Center of

Excellence, Janssen Pharmaceutical

Companies of Johnson \& Johnson

Welsh and McKean Roads, Spring

House, PA 19744, USA

e-mail:phornby@its.jnj.com
Physiologically relevant sources of absorptive intestinal epithelial cells are crucial for human drug transport studies. Human adenocarcinoma-derived intestinal cell lines, such as Caco-2, offer conveniences of easy culture maintenance and scalability, but do not fully recapitulate in vivo intestinal phenotypes. Additional sources of renewable physiologically relevant human intestinal cells would provide a much needed tool for drug discovery and intestinal physiology. We compared two alternative sources of human intestinal cells, commercially available primary human intestinal epithelial cells (hlnEpCs) and induced pluripotent stem cell (iPSC)-derived intestinal cells to Caco-2, for use in in vitro transwell monolayer intestinal transport assays. To achieve this for iPSC-derived cells, intestinal organogenesis was adapted to transwell differentiation. Intestinal cells were assessed by marker expression through immunocytochemical and mRNA expression analyses, monolayer integrity through Transepithelial Electrical Resistance (TEER) measurements and molecule permeability, and functionality by taking advantage the well-characterized intestinal transport mechanisms. In most cases, marker expression for primary hlnEpCs and iPSC-derived cells appeared to be as good as or better than Caco-2. Furthermore, transwell monolayers exhibited high TEER with low permeability. Primary hlnEpCs showed molecule efflux indicative of P-glycoprotein (Pgp) transport. Primary hlnEpCs and iPSCderived cells also showed neonatal Fc receptor-dependent binding of immunoglobulin $\mathrm{G}$ variants. Primary hlnEpCs and iPSC-derived intestinal cells exhibit expected marker expression and demonstrate basic functional monolayer formation, similar to or better than Caco-2. These cells could offer an alternative source of human intestinal cells for understanding normal intestinal epithelial physiology and drug transport.

Keywords: human intestinal epithelial cell (hInEpC), induced pluripotent stem cell (iPSC), permeability, Transepithelial Electrical Resistance (TEER), neonatal Fc receptor (FcRn)

\section{INTRODUCTION}

The use of in vitro cell models for human drug transport studies has focused on intestinal epithelial cells, as these cultures contain primarily absorptive cells. While isolated human intestinal epithelial cells (hInEpCs) retain important in vivo anatomical and biochemical features, they are difficult to culture and have limited viability. As a result, immortalized human adenocarcinoma cell lines have been extensively used to study absorption mechanisms. While immortalized cells offer many advantages, extrapolation of data generated with these cell lines to in vivo conditions is often difficult, as these cells originated from tumors and are therefore not representative of the true physiological environment (Le Ferrec et al., 2001). In addition, these cells form monolayers that are widely used for small molecule intestinal permeation in vitro studies (below). But, with increasing numbers of biotechnology protein therapeutics and novel scaffolds available, which open the possibility for oral delivery, there is a need for alternatives that more closely recapitulate the physiology of the intestinal epithelial cell.
The human colorectal adenocarcinoma cell line Caco-2 is frequently used for drug absorption studies, particularly in the context of small molecules (Le Ferrec et al., 2001; Balimane and Chong, 2005). Caco-2 cells are easy to culture and have the capacity to spontaneously differentiate into cells possessing the morphology and function of enterocytes, the absorptive cells of the intestine (Balimane and Chong, 2005). Caco-2 cells are commonly cultured on semi-permeable inserts in a transwell format, where the cells form a polarized monolayer (Leonard et al., 2000; Le Ferrec et al., 2001), and the transport of molecules between the apical and basolateral chambers can be easily evaluated. While Caco-2 cells are a good model for observation of passive transcellular and paracellular permeability (Balimane and Chong, 2005), there are differences in cytokine production and cytokine receptor expression between Caco-2 cells and normal epithelial cells (Aldhous et al., 2001). In addition, Caco-2 cells under-express transporters and metabolizing enzymes relative to in vivo tissue, potentially excluding mechanisms crucial for drug absorption studies (Balimane and Chong, 2005). 
Due to the limitations of immortalized intestinal cell lines, many studies have focused on the use of primary hInEpCs as a more physiologically relevant cell-based model (Perreault and Beaulieu, 1998; Aldhous et al., 2001; Ootani et al., 2009; Lahar et al., 2011). However, stocks of these cells are difficult to maintain due to limited donors and low viability in culture. Recently, commercial sources of primary hInEpCs were made available (Lonza; Walkersville, MD), which greatly increase the convenience of obtaining primary cell stocks. Commercial quality control data suggest that these primary hInEpCs have the capacity to form monolayers with tight junctions and express general epithelial markers, such as cytokeratins 8 and 18 (Bosch et al., 1988); however, little characterization has be done on their expression of intestinal cell type-specific markers or transport function. Other efforts to enable long-term culture of primary cells and enhance physiological conditions have led to the development of 3-dimensional (3D) models of the intestinal epithelium, which have focused on the use of primary intestinal stem cells and directed differentiation of pluripotent stem cells.

Stem cells have the capacity to self-renew and differentiate into the various cell lineages that make up specific tissue types. For example, intestinal stem cells are responsible for the self-renewal of the gut epithelium, and have been used in developing 3D intestinal models. Leucine-rich repeat-containing G protein-coupled receptor 5 (LGR-5)-positive stem cells can be isolated from primary intestinal tissue and grown as $3 \mathrm{D}$ intestinal organoids with crypt-villius physiology and culturing capacity up to 8 months (Sato et al., 2009). While 3D organoids derived from primary intestinal stem cells appear to possess physiologically relevant phenotypes, they cannot be used to assess classical functionality typically determined within 2-dimensional transwell cultures, such as the formation of monolayers with tight junctions and intestinal permeability and transport.

An additional source of human intestinal cells is possible through directed differentiation of pluripotent stem cells to intestinal cell lineages. The recent advent of human induced pluripotent stem cells (iPSCs) has provided a huge therapeutic potential as a tool for drug discovery, as patientspecific somatic cells can be reprogrammed into an embryonic stem cell-like state that can be directly differentiated to a specific cell type of interest for more physiologically relevant disease modeling. Induced human intestinal organoids (iHIOs) have recently been derived from iPSCs (Spence et al., 2010), which are capable of expressing epithelial and intestinal markers such as caudal type homeobox 2 (CDX2) (hindgut marker), E-Cadherin (cell-to-cell junction marker), and Villin (epithelial brush border marker). However, to our knowledge, the methods for differentiating these into a polarized epithelial monolayer similar to Caco-2 have not been reported.

In this study, we assessed expression of markers and functional activity of the newly commercially available primary hInEpCs and iPSC-derived intestinal cells compared to Caco-2 (Figure 1) in cell-based in vitro assays. We adapted our previously described 3D intestinal organogenesis to differentiation within transwells.
Intestinal marker expression, formation of monolayers with tight junction formation and functional molecule transport and binding were evaluated.

\section{MATERIALS AND METHODS \\ CELL CULTURE \\ Intestinal cells}

Human primary small intestinal epithelial cells (Lonza; Walkersville, MD) from 3 donors (Donor A: Lot \# 0000258132; Donor B: Lot \# 0000256741; Donor C: Lot \# 0000256744) were thawed and cultured in transwell inserts for 10-11 days in SmGM-2 media (Lonza), according to manufacturer's instructions. Caco-2 colorectal adenocarinoma-derived cells (ATCC; Manassas, VA) were cultured in transwell inserts for 14-21 days in Caco-2 media (10\% fetal bovine serum, 1X non-essential amino acids, $1 \mathrm{X}$ sodium pyruvate, and $6 \mathrm{mM}$ L-Glutamine in Dulbecco's Modified Eagle's Medium (DMEM) High Glucose, reagents from Life Technologies; Carlsbad, CA).

\section{iPSCs}

A1145A and B2198A (Johnson \& Johnson; Spring House, PA) were produced from human kidney-derived cells using retroviral (Takahashi et al., 2007) and modified mRNA (non-viral) reprogramming methods (Yakubov et al., 2010), respectively. C2198A and C2200B (Johnson \& Johnson; Spring House, PA) were produced from human umbilical tissue-derived cells by modified RNA reprogramming methods. D2043A (System Biosciences; Mountain View, CA) was derived from human foreskin fibroblasts by retroviral methods. iPSC lines were maintained in mTeSR1 culture media (STEMCELL Technologies; Vancouver, BC) on culture dishes/flasks coated with Geltrex (Life Technologies) and passaged by Dispase (STEMCELL Technologies) dissociation every 3-5 days as previously described (McCracken et al., 2011).

\section{iPSC DIRECTED DIFFERENTIATION Transwell differentiation}

Semi-permeable transwell inserts were coated apically with Geltrex (Life Technologies) prior to plating iPSCs within 12well transwell tissue culture plates. iPSCs were plated apically as cell clumps (5-10 cells per clump) at a density of 3000-6000 clumps per transwell insert. Two to three days after plating, iPSCs were differentiated within transwell inserts modified previously reported methods (Spence et al., 2010) into definitive endoderm using GDF8 with GSK3b inhibitor and B27 supplement then differentiated to hindgut using Keratinocyte Growth Factor and Retinoic Acid then EGF, Noggin, and R-Spondin 1 for $>26$ days (Kauffman et al., Submitted) on both sides of the transwell insert; however, the many spheroid structures observed at Stage 2 were maintained as part of the adherent layer throughout Stage 3 differentiation (Table $\mathbf{1}$ ) for up to 31 days.

\section{MONOLAYER ASSESSMENT}

\section{Transepithelial electrical resistance (TEER)}

TEER of transwell cultures was recorded in measurements of Ohms using an Epithelial Volt Ohm Meter (EVOM) ${ }^{2}$ and electrode set (World Precision Instruments; Sarasota, FL). Raw 


\title{
Intestinal \\ Carcinoma \\ $\left\lfloor\begin{array}{l}\text { Tissue } \\ \text { Dissociation }\end{array}\right.$ \\ Normal \\ Intestinal Tissue \\ Induced Pluripotent \\ Stem Cell Line
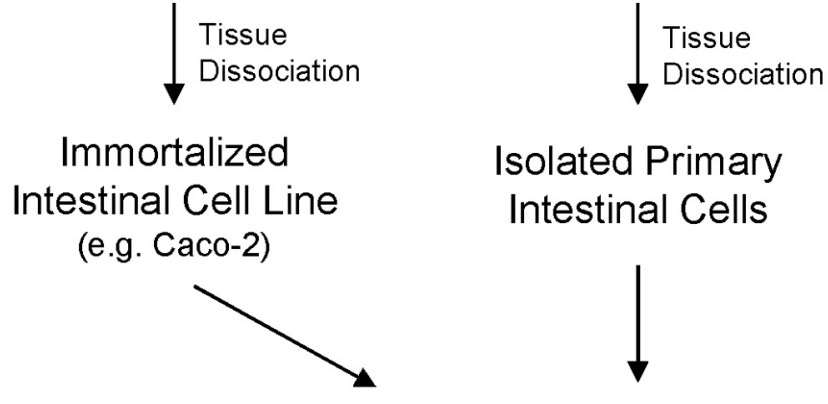 \\ Isolated Primary Intestinal Cells
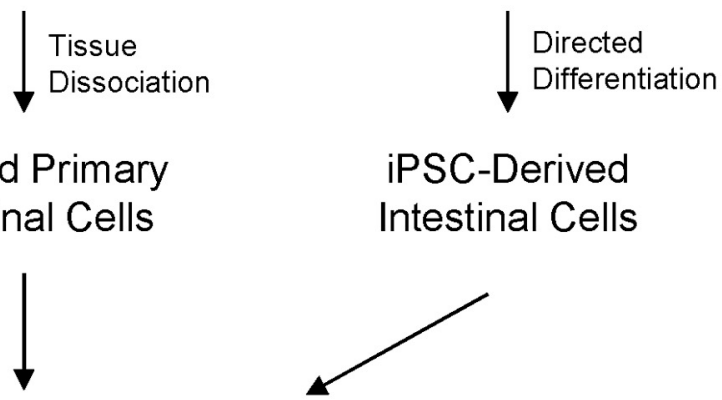 \\ iPSC-Derived Intestinal Cells \\ In Vitro Intestinal Epithelial Cell Transwell Culture
}

\author{
$\downarrow$ \\ Development of Physiologically \\ Relevant Phenotypes \\ Monolayer Morphology \\ Tight Junction Formation \\ Intestinal Marker Expression \\ Transport Function
}

FIGURE 1 | Overview of isolation and utilization of human intestinal epithelial cells in transwell cultures for in vitro assays. Major sources of human intestinal cells include intestinal carcinoma tissue, normal intestinal tissue, or directed differentiation of induced pluripotent stem cells.

Table 1 | Summary of growth factors and differentiation cell culture conditions used for transwell differentiation, relative to previously established intestinal organogenesis to 3D organoids.

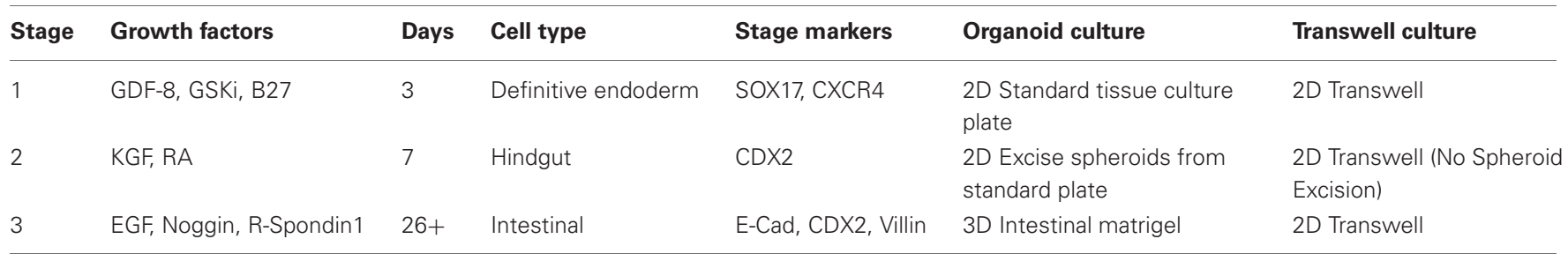

data was converted to $\Omega \times \mathrm{cm}^{2}$ based on area of transwell plate inserts $\left(1.12 \mathrm{~cm}^{2}\right)$.

\section{FITC-dextran permeability}

Cells in 24-well transwell plates were washed twice with DPBS (Life Technologies). To the apical side, $200 \mu \mathrm{L}$ of $12.5 \mathrm{mg} / \mathrm{mL}$ Fluorescein isothiocyanate-dextran, molecular weight $150 \mathrm{kDa}$ (FD150) (Sigma-Aldrich) diluted in Caco-2 media $(p H=6.0)$ was. To the basolateral side, $500 \mathrm{uL}$ of Caco- 2 media $(p H=7.4)$ was added. After a 90 -min incubation at $37^{\circ} \mathrm{C}, 100 \mathrm{uL}$ of media was collected from the basolateral chamber and analyzed for the presence of FITC-Dextran using a SpectraMax M5 microplate reader (Molecular Devices; Sunnyvale, CA).

\section{P-GLYCOPROTEIN TRANSPORT ASSAY}

Primary hInEpCs were dosed on the apical or basolateral side with a $2 \mathrm{mM}$ mix of Digoxin and Atenolol in HBSSg [2 mM glucose and $10 \mathrm{mM}$ 4-(2-hydroxyethyl)-1-piperazineethanesulfonic acid in Hank's Balanced Salt Solution (HBSS)] with calcium and magnesium, $p H=7.4$ ) and incubated for $90 \mathrm{~min}$ at $37^{\circ} \mathrm{C}$ in the presence or absence of $10 \mu \mathrm{M}$ Cyclosporin A (CSA). Samples were collected from both the apical/donor and basolateral/receiver chambers and analyzed by LCMS. Digoxin and Atenolol levels in each condition were used to calculate apparent permeability $\left.(P a p p=\delta C r / \delta t) \times V r /\left(A \times C_{0}\right)\right)$ in the apical to basolateral $(\mathrm{A}-\mathrm{B})$ or basolateral to apical $(\mathrm{B}-\mathrm{A})$ direction. $\delta \mathrm{Cr}=$ final receiver concentration; $\delta \mathrm{t}=$ assay time; $\mathrm{Vr}=$ receiver volume; 
$\mathrm{A}=$ transwell growth area; $\mathrm{C}_{0}=$ initial apical concentration. To ensure monolayer integrity during the assay, all wells were dosed apically with $100 \mu \mathrm{g} / \mathrm{mL}$ Lucifer Yellow (LY) at the start of Digoxin and Atenolol incubation, and samples were collected from the basolateral chamber for analysis by SpectraMax M5 microplate reader at the end of the 90 min incubation. Only transwells with a LY Papp $(\mathrm{A}-\mathrm{B})$ of $<1 \times 10^{-6} \mathrm{~cm} / \mathrm{s}$ were used in calculating final Papp Ratios for Digoxin and Atenolol. Based on this cutoff, one of 8 wells each for hInEpC Donors A and $\mathrm{B}$, and two of 8 wells for Donor $\mathrm{C}$ was excluded from data analysis.

\section{MRNA EXPRESSION}

RNA was harvested from Caco-2, primary hInEpCs, or iPSCs before or after differentiation to Stage 3 by RNeasy Mini kit (Qiagen; Germantown, MD), and reverse transcribed to cDNA using the $\mathrm{RT}^{2}$ First Strand kit (Qiagen). Using $30 \mathrm{ng} / \mu \mathrm{L}$ starting cDNA, samples were used in reactions within Custom $\mathrm{RT}^{2}$ Profiler PCR array containing probes supplied by the manufacturer (SABiosciences; Valencia, CA) for intestinal and control markers (Table 2), following reaction cycling conditions outlined in the manufacturer's protocol. Data analysis was performed using the $\Delta$ CT method, where raw $C t$ values were normalized to housekeeping gene $60 \mathrm{~S}$ acidic ribosomal protein P0 (RPLP0) before comparing expression relative to Caco-2. Expression levels for primary hInEpCs represents the average across all three donors used in this study.

Table 2 | List of probes used in Custom $\mathrm{RT}^{2}$ Profiler PCR array mRNA expression analyses of intestinal and control markers in intestinal epithelial cells.

\begin{tabular}{lll}
\hline Gene & NCBI reference no & SAB catalog no \\
\hline E-Cadherin & NM_004360 & PPH00135 \\
CDX2 & NM_001265 & PPH13618 \\
KLF5 & NM_001730 & PPH00434 \\
Villin & NM_007127 & PPH23365 \\
SOX9 & NM_000346 & PPH02125 \\
LGR5 & NM_003667 & PPH13346 \\
ASCL2 & NM_005170 & PPH12852 \\
MUC2 & NM_002457 & PPH06990 \\
Chromogranin A & NM_001275 & PPH01181 \\
LYZ & NM_000239 & PPH14748 \\
VIM & NM_003380 & PPH00417 \\
FCRn & NM_004107 & PPH11194 \\
CXCR4 & NM_003467 & PPH00621 \\
PDX1 & NM_000209 & PPH05536 \\
OCT4 & NM_002701 & PPH02394 \\
TNNT2 & NM_000364 & PPH02619 \\
PAX6 & NM_000280 & PPH02598 \\
TUBB3 & NM_006086 & PPH02607 \\
RPLP0 & NM_001002 & PPH21138 \\
\hline PrObes for the &
\end{tabular}

Probes for the NCBI Reference sequence numbers listed were obtained from SABiosciences $(S A B)$ using the catalog numbers provided.

\section{PROTEIN EXPRESSION}

\section{Flow cytometry}

Undifferentiated or Stage 1 iPSCs were detached by treatment with Accutase (Sigma-Aldrich; St. Louis, MO) and stained for viability by Near Infrared Live/Dead kit (Invitrogen; Carlsbad, CA) before fixation in Cytofix Buffer (BD Biosciences; San Jose, CA). For pluripotent marker analyses, cells were stained using Human Pluripotent Stem Cell Transcription Factor Analysis or Human Pluripotent Stem Cell Sorting and Analysis kits (BD Biosciences). For definitive endoderm marker analysis, cells were stained with a 1:5 dilution of PE-conjugated mouse anti-human CD184/ C-X-C chemokine receptor type 4 (CXCR4) (306506, Biolegend; San Diego, CA) before permeabilized with Phosflow Perm Buffer (BD Biosciences) and stained with a 1:5 dilution of APC-conjugated goat polyclonal anti-human Sry-related HMG box 17 (SOX17) (IC1924A, R\&D Systems; Minneapolis, $\mathrm{MN})$. For analysis of surface expression of neonatal Fc receptor $(\mathrm{FcRn}), \mathrm{Caco}-2$ and primary hInEpCs were dissociated from transwell culture by Accutase, incubated with $50 \mu \mathrm{g} / \mathrm{mL}$ affinity purified rat anti-human FcRn polyclonal antibody (generated in house), followed by incubation with $7.5 \mu \mathrm{g} / \mathrm{mL}$ FITC Donkey anti-rat IgG (FAb2) secondary antibody (109-006006, Jackson Immunoresearch; West Grove, PA). Fluorescence of stained cells was measured in conjunction with appropriate compensation controls (BD Biosciences) by flow cytometry using an LSR Fortessa FACS Sorter (BD Biosciences). Raw data was analyzed by FlowJo analysis software (Tree Star; Ashland, OR), with gating parameters set based on isotype controls (Figure A1A).

\section{Immunofluorescence}

iPSC-derived cells Stage 2 iPSC-derived cells were fixed in 4\% paraformaldehyde (Electron Microscoopy Sciences; Hatfield, PA) before permeabilization with $0.5 \%$ Triton X-100 (Electron Microscoopy Sciences). After addition of Image-iT ${ }^{\circledR}$ FX signal enhancer (Invitrogen), cells were treated with 1X Blocking Buffer (Sigma) before exposure to a 1:50 dilution of ECadherin, CDX2, or Villin antibodies (Dako), using manufacturer recommended concentrations. Immunoreactivity to primary antibodies was detected with a 1:500 dilution of AF568conjucated goat anti-mouse antibody (Invitrogen). Prolong GOLD antifade with DAPI (Invitrogen) was added to wells prior to visualizing on a Nikon SMZ-1500 fluorescence dissecting microscope.

\section{FcRn-DEPENDENT IMMUNOGLOBULIN G (IgG) BINDING}

Caco-2, hInEpCs (Donor A), or iPSC-derived cells were detached from transwells by Accutase (Sigma) treatment and washed twice with Dulbecco's Phosphate-Buffered Saline (DPBS) (Life Technologies). Cells were transferred in DPBS to MesoScale Discovery (MSD) High Bind plates (MSD; Rockville, MD) at a density of $1 \times 10^{4}$ or $2.5 \times 10^{4}$ cells per well, and incubated at room temperature for $2 \mathrm{~h}$ to allow attachment to the plate surface. Plates were then blocked with 20\% Fetal Bovine Serum (Life Technologies) and $0.18 \%$ Sodium Azide (VWR International; Radnor, PA) for $15 \mathrm{~min}$ at room temperature. Wells were washed once with DPBS at $p H 6.0$ before incubation with FcRn-binding 
variants (anti-RSV N434A or anti-RSV H435A in DPBS at $p \mathrm{H}$ 6.0) for $90 \mathrm{~min}$ at $37^{\circ} \mathrm{C}$. Plates were washed 3 times with DPBS at $p H$ 6.0, and cells were incubated with ruthenium-labeled goat anti-human IgG $\mathrm{F}\left(\mathrm{ab}^{\prime}\right) 2(1 \mu \mathrm{g} / \mathrm{ml}$ in DPBS at $p \mathrm{H} 6.0)$ for $1 \mathrm{~h}$ at room temperature. Cells were washed 3 times with DPBS at $p \mathrm{H}$ 6.0. Tris-based Read Buffer $\mathrm{T}$ without surfactant (MSD) was added to wells immediately before measuring Relative Luminscent Units (RLU) using a Sector Imager 6000 reader and Discovery Workbench software (MSD). For analysis, background signal RLUs were subtracted from RLUs of samples run in triplicate.

\section{RESULTS \\ MULTIPLE SOURCES OF HUMAN INTESTINAL EPITHELIAL CELLS EXHIBIT INTESTINAL MARKER EXPRESSION}

Along with limited viability in culture, one of the major drawbacks of routinely using primary hInEpCs for studying intestinal physiology is that these cells must be collected from human donors. A newly available commercial source of primary hInEpCs greatly increases the convenience of obtaining primary cell stocks, but need to be further characterized for intestinal cell-type specific marker expression and functional monolayer formation. Thus, we sought to characterize these commercially available stocks to assess their physiological relevance for use in cell-based in vitro assays of intestinal uptake and transport.

As previously reported, a measure of intestinal cell phenotypic quality is the expression of a panel of general intestinal epithelial markers (Spence et al., 2010). We used immunocytochemistry to verify expression and expected localization of several representative intestinal epithelial markers within primary hInEpCs from three different donors (Figure 2, top row; Figure A1B), relative to the immortalized human intestinal epithelial cell line Caco-2
(Figure 2, bottom row). This was visualized for E-cadherin, an epithelial cell adhesion marker found within tight junctions between cells (Zbar et al., 2004), CDX2, a transcription factor that is upstream of signaling promoting intestinal cell fate (Gao et al., 2009) and Villin, an actin-binding protein associated with the intestinal brush border of absorptive enterocytes (Friederich et al., 1999). The localization of these markers within cells was appropriate in primary hInEpCs and Caco-2 (Figure 2). Marker expression intensity was variable between the three hInEpC donors within this study, with strongest expression evident in Donor A (Figure 2, top row; Figure A1B). Expression results for hInEpCs and Caco-2 were used to compare to iPSC-derived iHIOs grown in transwells (below).

In order to evaluate iPSC-derived intestinal cell phenotypes within classical in vitro monolayer conditions, we adapted intestinal organogenesis to transwell culture by performing iPSC differentiation through Stage 3 within transwell inserts (Table 2, Figure 3A). Differentiation of a panel of iPSC lines (A1145A, B2198A, C2128A, C2200B, and D2043A) on matrigel-coated transwell inserts using Myostatin, Glycogen Synthase Kinase $3 \beta$ inhibitor and B27 supplement resulted in cells expressing definitive endoderm markers SOX17 and CXCR4 (Figure 3B), similar to iPSCs differentiated to Stage 1 in standard tissue culture plates (Kauffman et al., Submitted). Further differentiation of iPSC-derived definitive endoderm cells within transwell plates, by addition of Keratinocyte Growth Factor and Retinoic Acid, produced layers of cells with developing spheroid structures at Stage 2 in many of the iPSC lines (Figure 3C, top row). CDX2 expression at Stage 2 was relatively uniform within layers of cells differentiating in transwells (Figure 3C, bottom row). However, D2043A showed more intense expression around spheroid structures.
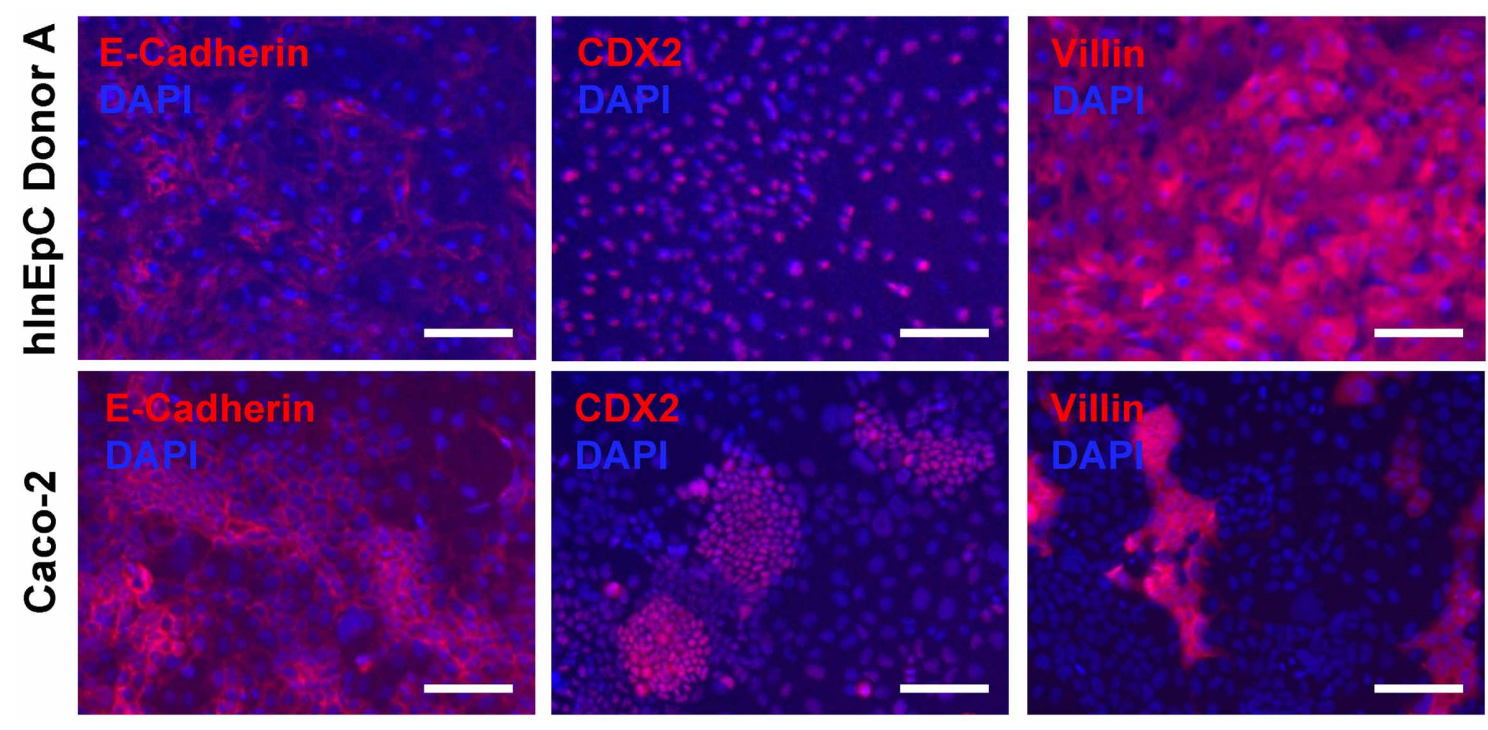

FIGURE 2 | Characterization of intestinal marker expression in commercially available (Lonza) primary human intestinal epithelial cells (hlnEpCs) relative to immortalized human intestinal cell line Caco-2. Top row: primary hlnEpCs uniformly express intestinal epithelial markers
E-Cadherin (tight junctions), CDX2 (hindgut), and Villin (enterocytes). Primary hlnEpC Donor A shown (see Figure A1B for Donors B and C). Bottom row: Caco-2 show less uniform expression of enterocyte marker Villin. Scale bar, $100 \mu \mathrm{m}$. 


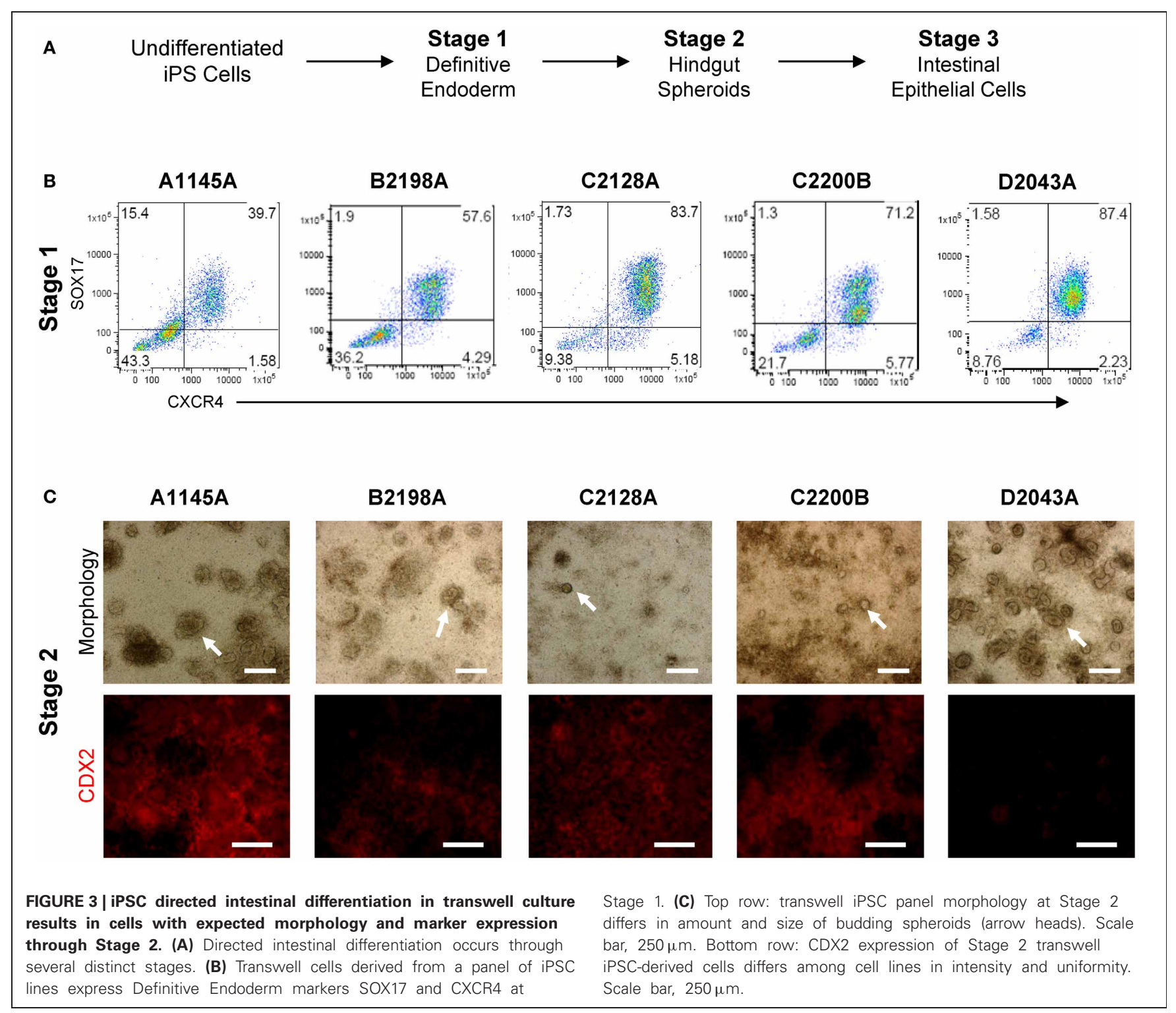

iPSC-derived Stage 3 3D intestinal organoids exhibit E-Cadherin, CDX2, Villin, and Chromogranin A immunoreactivity with the expected localization, (Kauffman et al., Submitted). In this study, a supply of transwell A1145A iPSCderived Stage 3 Day 31 cells was limited, and did not allow sufficient material for extensive immunocytochemistry analyses of intestinal markers. Thus, mRNA expression analysis was used to directly compare larger panel of known intestinal marker and differentiation control genes Stage 3 A1145A iPSC-derived intestinal marker expression to Caco-2 and primary hInEpCs by RT-PCR (Figure 4). Differentiated Stage 3 iPSCs showed increased marker expression relative to undifferentiated cells, usually reaching a level more similar to primary hInEpCs in the case of 3D iHIOs (Figure 4), or more similar to Caco-2 in the case of transwell-differentiated iPSCs (Figure 4). Intestinal markers that followed this expression pattern included epithelial tight junction marker E-Cadherin (Zbar et al., 2004), hindgut epithelial marker CDX2 (Gao et al., 2009), enterocyte maker Villin (Friederich et al., 1999), enteroendocrine marker Chromogranin A (O'Connor et al., 1983), and Mucin-2 (MUC2), a marker for intestinal goblet cells (Gum et al., 1999) (Figures 4A-E).

For some intestinal makers, expression in iPSC-derived intestinal cell types was highest in iPSC-derived cells. For example, expression levels of intestinal crypt cell marker Sex determining region Y-box 9 (SOX9), paneth cell marker Lyzosyme (LYZ) (Peeters and Vantrappen, 1975), and intestinal stem cell marker LGR5 (Barker et al., 2007) were greatest in iHIOs and transwell differentiated cells compared to Caco-2 or primary hInEpCs (Figures 4F-H). On the other hand, an additional intestinal stem cell marker, Achaete Scute-Like 2 (ASCL2) (van der Flier et al., 2009), was most highly expressed in Caco-2 (Figure 4I). In the case of intestinal epithelial cell differentiation transcription factor Kruppel-like factor 5 (KLF5) (Bell et al., 2013), mRNA expression 

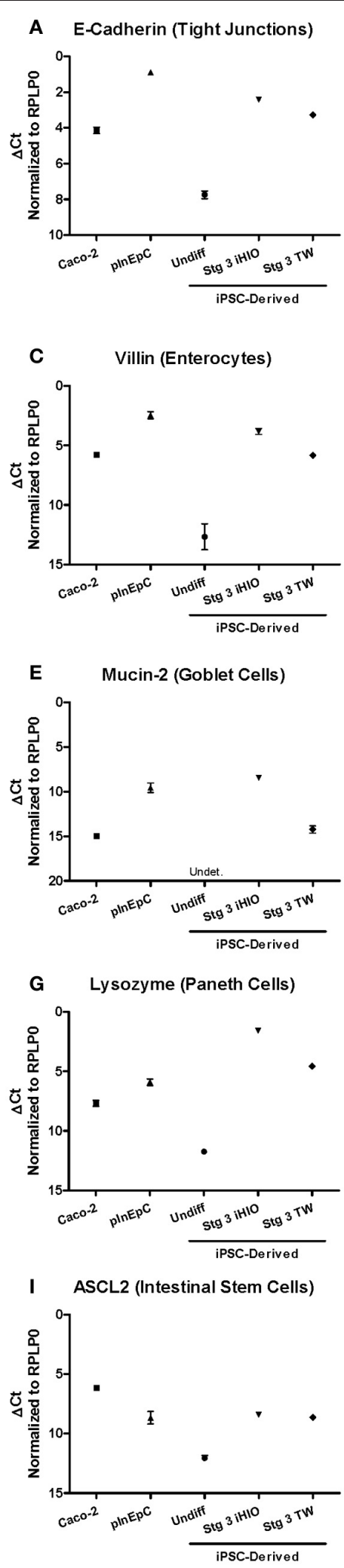

FIGURE 4 | Comparison of intestinal marker mRNA expression in multiple sources of human intestinal epithelial cells. Expression of tight junction marker (A) and hindgut epithelia (B), as well as specific epithelial lineage markers (C-J) intestinal markers of epithelial intestinal lineages was evaluated by RT-PCR in Caco-2, primary hlnEpCs (average

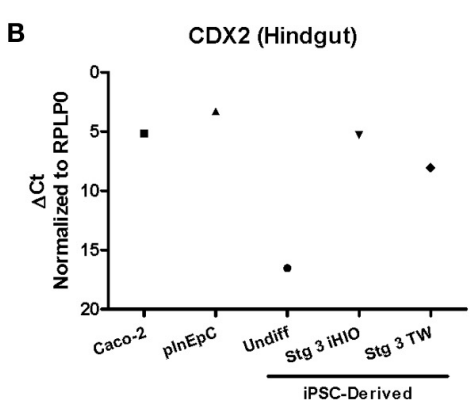

D Chromogranin A (Enteroendocrine Cells)

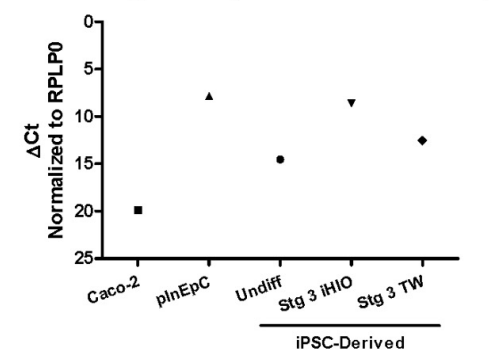

$\mathbf{F}$

sox9 (Crypt Cells)

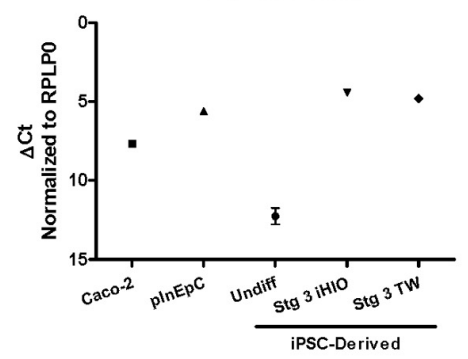

H

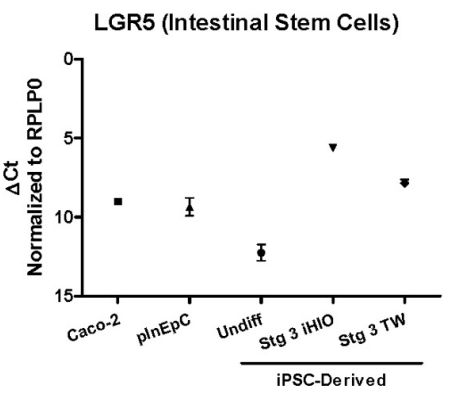

$\mathbf{J}$ KLF5 (Hindgut)

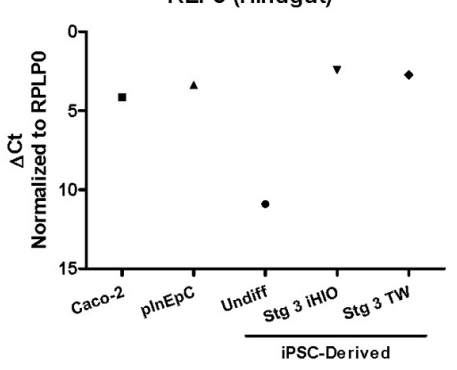

of Donors $\mathrm{A}-\mathrm{C}$ ), and iPSC-derived undifferentiated (Undiff), Stage 3 induced human intestinal organoids (Stg $3 \mathrm{iHIOs),} \mathrm{or} \mathrm{Stage} 3$ transwell intestinal cells (Stg $3 \mathrm{TW}$ ). Using the $\Delta \mathrm{Ct}$ method, raw $\mathrm{Ct}$ values were normalized to housekeeping gene RPLPO. $N=3$; Error bars represent SEM. 
levels were similar for all intestinal cell types (Figure 4J). The expression of several control genes [e.g., pluripotent marker Octamer-binding Transcription factor 4 (OCT4) (Nichols et al., 1998)] confirmed the cell sample quality (Figure A3).

\section{PRIMARY hInEpCs AND iPSC-DERIVED INTESTINAL CELLS FORM TRANSWELL MONOLAYERS WITH TIGHT JUNCTIONS}

While all three primary hInEpCs donors appeared to be capable of forming confluent cell layers in transwell culture that expression the tight-junction marker E-Cadherin (Figures 2, 4A), we assessed tight junction formation functionally through measurements of Transepithelial Electrical Resistance (TEER) and monolayer permeability. As confluent epithelial monolayers form, TEER measurements generally increase, reaching 260-420 $\Omega \times \mathrm{cm}^{2}$ on average in Caco-2 cultures (Le Ferrec et al., 2001). We found that after 11 days of transwell culture, primary hInEpCs from all three donors exhibited TEER measurements of $>1500 \Omega \times \mathrm{cm}^{2}$ (Figure 5A), providing a strong indication of tight junction formation. As changes in epithelial TEER can also be explained by changes in transcellular ion permeability (Yu and Sinko, 1997), we confirmed tight junction formation of pInEpC transwell cultures by determining the monolayer permeability to FITC-labeled Dextran at a molecular weight of 150,000 (FD150). Apical side incubation in transwell chambers, resulted in $<3 \%$ FITC-labeled Dextran detected on the basolateral side, relative to control transwells with no cells for primary hInEpC Donors A and B (Figure 5B). Donor C, which corresponds to the cells with highly variable TEER measurements (Figure 5B) showed slightly more FITC-Dextran permeability $(\sim 3 \%$ relative to control transwells).

While Stage 3 Day 31 transwell A1145A iPSC-derived cells exhibited intestinal marker expression consistent with other intestinal epithelial cell sources, it was difficult to gauge at which point during Stage 3 differentiation iPSC-derived cells may begin to take on functional phenotypes. Thus, we performed a time course experiment in which A1145A iPSCs were differentiated within transwell culture and assessed for monolayer morphology and evidence of tight junction formation throughout Stage 3 (Days 0, 7, 14, 21, 31). Morphologically, A1145A iPSC-derived cells showed 3D structures and dense patches of cells within transwells at the beginning of Stage 3 that appeared to disappear as differentiation progressed, leaving a flat monolayer-like layer of cells by Day 31 of Stage 3 (Figure 5C). To assess tight junction formation in confluent A1145A iPSC-derived transwell monolayers, we measured TEER and FITC-Dextran permeability during iPSC transwell differentiation. At Day 0 of Stage 3 differentiation, A1145A iPSC-derived cells within transwells exhibited low TEER measurements of less than $200 \Omega \times \mathrm{cm}^{2}$ which steadily increased to measurements reaching $937 \Omega \times$ $\mathrm{cm}^{2}$ by Day 31 (Figure 5D). At Stage 3 Day 0, iPSC-derived cells showed FD150 permeability of $\sim 2 \%$ relative to the no cell control (Figure 5E), a range similar to that seen for primary hInEpCs (Figure 5B). Coinciding with changes in TEER measurements during differentiation (Figure 5D), FD150 permeability decreased for iPSC-derived cells to as low as $0.06 \%$ of the no cell control (Figure 5E).

\section{INITIAL ASSESSMENT OF INTESTINAL EPITHELIAL TRANSPORT FUNCTION}

To further validate these cell sources functionally, we assessed monolayers for molecule transport or binding. A previously established mechanism influencing small molecule transport by intestinal epithelial cells is efflux by membrane associated ATPbinding cassette P-glycoprotein (Pgp) transporters, which facilitate cellular efflux to prevent accumulation of their substrates (Murakami and Takano, 2008). To assess Pgp transport activity for a given substrate, the basolateral to apical (B-A) permeability is compared to apical to basolateral (A-B), where compounds with efflux ratios $(\mathrm{B}-\mathrm{A} / \mathrm{A}-\mathrm{B})$ greater than 2 or 3 are generally considered to be Pgp substrates (Balimane et al., 2006). Using transwell monolayers of primary hInEpCs, we found that efflux ratios for Digoxin, a compound known to be highly effluxed by Pgp (Balimane et al., 2006), were $>8$ for pInEpC Donors A and $\mathrm{B}$ (Figure 6A). Importantly, efflux of Digoxin was reduced when these cells were also treated with CSA, a known Pgp transporter inhibitor (Watanabe et al., 1997). Atenolol, a poorly Pgp-effluxed compound (Balimane et al., 2006) showed very low efflux ratios of $<1$ in all three primary hInEpC donors, which was not further reduced by CSA (Figure 6B). During the Pgp transport assay, transwells were also dosed apically with lucifer yellow to confirm primary hInEpC monolayer integrity based on permeability of this fluorescent molecule (Figure A3). While the average apparent permeability for all three primary hInEpC donors was below $1.5 \times 10^{-6} \mathrm{~cm} / \mathrm{s}$, only transwells below this standard cutoff were used for analysis of Digoxin and Atenolol flux.

Unfortunately, as A1145A iPSC-derived transwell intestinal cells were very limited, there were insufficient cells within this study to perform a properly-controlled Pgp transport assay. Thus, to further validate iPSC-derived cells, we assessed FcRn immunoreactivity and performed a cell surface binding assay for neonatal Fc receptor ( $\mathrm{FcRn})$-mediated transport, which was amenable to the limited cell supply.

Intense FcRn expression in iPSC-derived intestinal organoids was noted as intense single-cell expression within the population of cells (Figure A4A) similar that previously reported for human intestinal tissue (Dickinson et al., 1999). We confirmed FcRn expression immunocytochemically in transwell monolayer cultures of Caco-2 and primary hInEpCs both intracellularly (Figure 6C) and on the surface of cells (Figure A4B). Real-Time RT-PCR analysis showed that FcRn mRNA expression increases to the level close to that of Caco-2 by Stage 3 of transwell differentiation (Figure 6D), whereas FcRn expression was highest in primary hInEpCs and iHIOs.

We examined binding of high or low FcRn-binding IgG mAb variants to Caco-2, primary hInEpCs, or iPSC-derived transwell intestinal cells by Meso Scale Discovery assay. Use of this highly sensitive ELISA-like assay allowed us to perform FcRn-dependent binding experiments with proper controls, even with limitedsupply iPSC-derived cells. Similar to Caco-2, primary hInEpCs showed significantly higher binding of the IgG variant with a high affinity to FcRn (N434A) than the low FcRn-binding variant (H435A) (Figure 6E). iPSC-derived intestinal cells also demonstrated significantly higher binding of a high FcRn-binding IgG variant (M428L) relative to the low FcRn-binding variant 


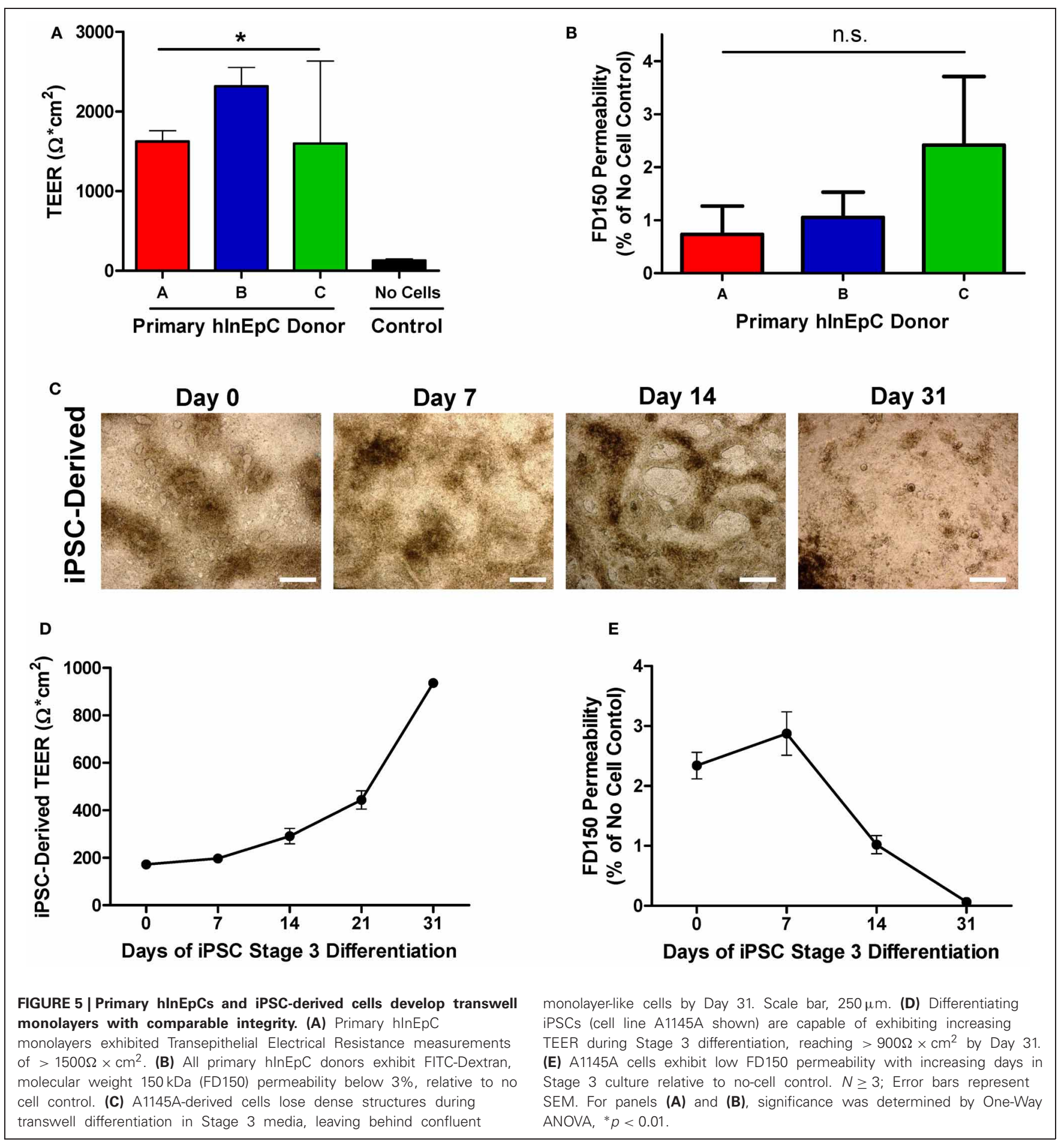

(H435A), however, maximal cell binding was not as high as Caco-2 (Figure 6F).

\section{DISCUSSION}

The major findings of this study are that marker expression of primary hInEpCs and iPSC-derived intestinal cells were on the same order of magnitude, or better than Caco-2, as determined by immunocytochemistry and mRNA expression analyses. The
iPSC-derived intestinal cells were successfully adapted to differentiation within 2D transwell monolayer culture, and similar to primary hInEpCs, demonstrated functional tight junctions with TEER and low permeability similar to, or better than, Caco-2. Initial assessment suggested functional activity for intestinal transporters such as Pgp transport (primary hInEpCs) or FcRn-dependent binding of molecules (primary hInEpC and iPSC-derived cells). The main conclusions from our study are 


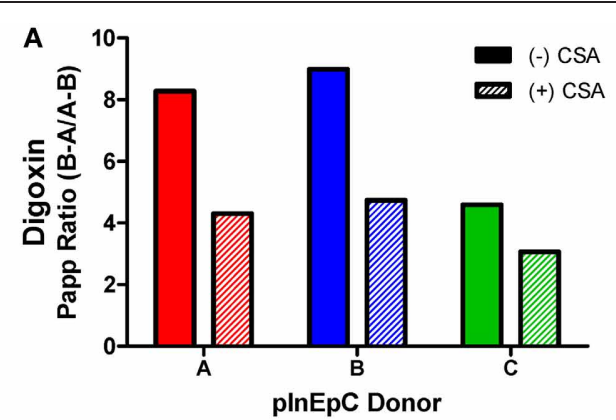

C

\section{Caco-2}
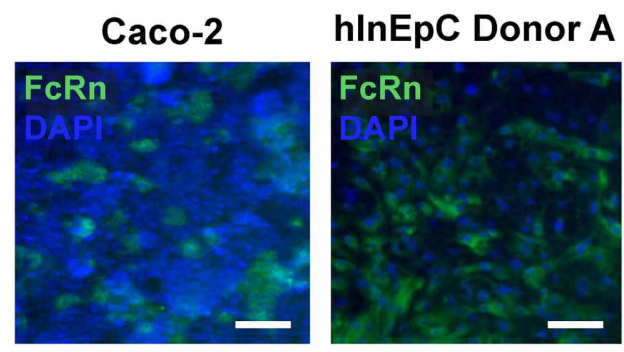

E

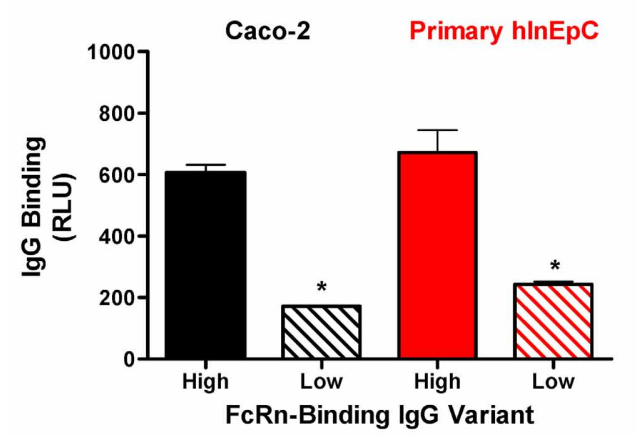

FIGURE 6 | Primary hlnEpCs and iPSC-derived intestinal monolayers show functional activity in transwell culture. (A) plnEpC donors $A$ and $B$ show Digoxin Papp Ratios > 8, which are decreased in the presence of cyclosporin A (CSA), suggesting that these cell lines have P-glycoprotein (Pgp) transport activity. (B) Atenolol Papp ratios for all samples fell below the expected $<2$ cutoff for Pgp activity. (C) Primary hlnEpCs (right) show more uniform intracellular FcRn expression than Caco-2 (left), as shown by immunofluorescence. Scale bar, $100 \mu \mathrm{m}$. (D) iPSC-derived transwell cells

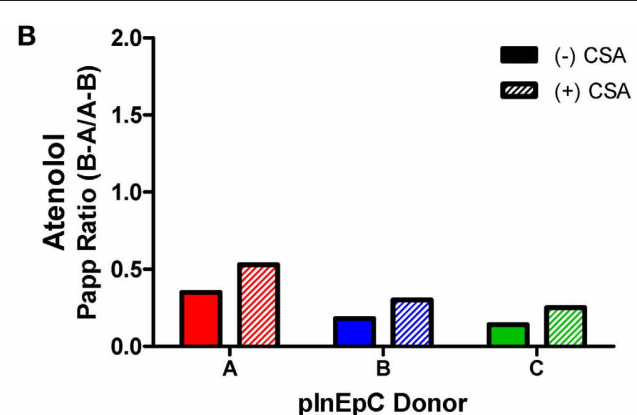

D

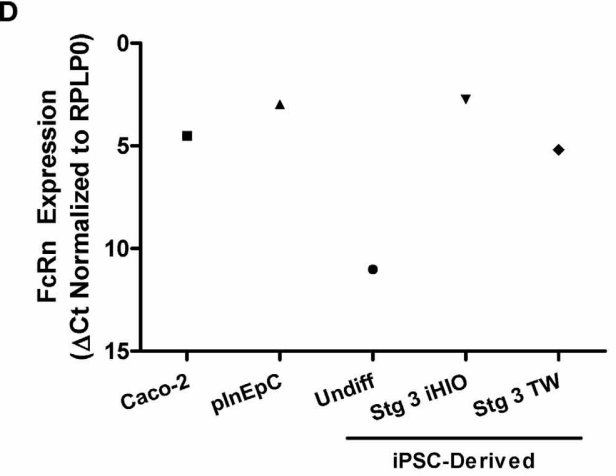

$\mathbf{F}$

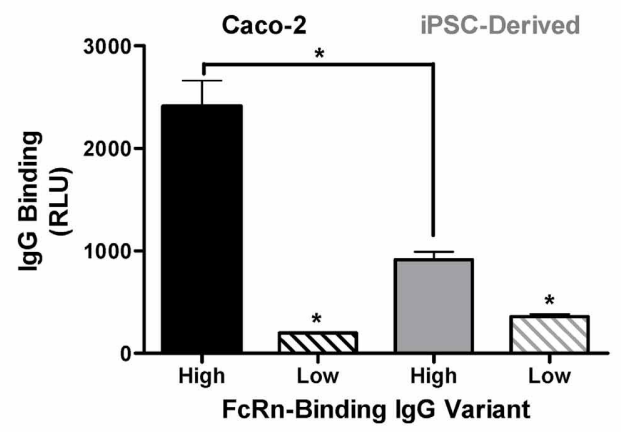

express FcRn mRNA levels similar to Caco-2, but lower than primary hlnEpCs and iPSC-derived 3D induced human intestinal organoids (iHIOs). Caco-2 (E,F), primary hInEpCs (E) and iPSC-derived transwell cells (F) show

FcRn-dependent mAb binding by MSD assay, as demonstrated by statistically greater cell binding of high FcRn-binding IgG variants [High, N434A (E) or M428L (F)] than low FCRn-binding variants (Low, H435A). N $\geq 4$; Error bars represent SEM. (E,F), statistical significance determined by $t$-test, $* p<0.001$. further discussed below in relation to previously published studies.

Before using a recently released commercially available source of primary hInEpCs for comparing iPSC-derived intestinal cells, it was necessary to more fully characterize this intestinal epithelial cell source for marker expression and barrier function. The extent to which primary hInEpCs expressed markers by immunofluorescence varied, with expression of E-Cadherin, Villin, and CDX2 strongest in Donor A; however, intestinal marker expression appeared to be more consistent between donors by RT-PCR analysis. While all three donors exhibited high TEER $\left(>1500 \Omega \times \mathrm{cm}^{2}\right)$, TEER and FD150 permeability showed high variability in Donor C. Moreover, Donor C showed poor efflux of Pgp-transported Digoxin, suggesting that this primary hInEpC donor is less useful for intestinal barrier function and transport than Donors $A$ and $B$.

A 3D directed intestinal organogenesis protocol was successfully adapted to 2D transwell differentiation, based on intestinal marker similarity. Stage 1/Definitive endoderm markers SOX17 and CXCR4 were expressed in $40-87 \%$ of the cell population. Stage 2-marker CDX2 was also robustly expressed within 
transwell cultures. Stage 3 differentiation within transwells of iPSC-line A1145A (Kauffman et al., Submitted) was similar to the development of iHIOs, except that spheroid structures that formed by Stage 2 of differentiation were not excised for $3 \mathrm{D}$ culture, but allowed to continue differentiation within the adherent layer of cells. Interestingly, spheroid structures found at Stage 2 were gradually lost, so that by Stage 3, Day 31, a monolayer-like morphology was found within transwell cultures, which showed TEER measurements more similar to primary hInEpCs and greater than the average TEER for Caco-2 transwell cultures (Le Ferrec et al., 2001). Consistent with this result, low permeability of FD150 for primary hInEpCs and iPSC-derived transwell cells suggests that a barrier function was present in these cell cultures. It is important to note that the TEER obtained in this study for human intestinal epithelial cell sources is several-fold higher than typically found in intestinal tissue ex vivo; however, high TEER values may not discount the physiological relevance of these cells in vitro, as the presence of several epithelial layers and cell types within intestinal tissue can add multiple impedances to intestinal transmural TEER measurements (Gitter et al., 1998, 2000).

In addition to A1145A, which shows consistent robust intestinal differentiation in $3 \mathrm{D}$ and $2 \mathrm{D}$ monolayer culture, we also performed a transwell differentiation time course on C2128A because this cell line showed uniform expression of CDX2 at Stage 2 within transwell culture, but demonstrated a distinct morphology. At Stage 2, C2128A cells appeared to already exhibit a monolayer-like morphology with little to no dense structures; therefore, we reasoned that C2128A might be more amenable to the development of iPSC-derived intestinal cells for use in functional transwell monolayer assays. However, as Stage 3 differentiation of C2128A-derived progressed, holes appeared within the layer of cells, which corresponded to low TEER measurements and high FD150 permeability at Stage 3 (Figure A5). Thus, differences in iPSC line intestinal differentiation capacity appear to be evident during 2D transwell culture differentiation.

mRNA expression analysis of an extensive panel of intestinal marker and control genes enabled a direct comparison of primary hInEpCs and iPSC-derived cell maker expression to Caco-2 cells. As expected, undifferentiated iPSCs showed levels of marker expression several fold lower than all intestinal samples with the exception of the pluripotent cell control marker OCT4 (Nichols et al., 1998). Differentiated iPSC-derived iHIOs and transwell cells exhibited upregulated intestinal marker expression that was more similar to primary hInEpCs than Caco-2, consistent with a better in vitro representation of human intestinal epithelia than Caco-2.

As might be expected, expression of definitive endoderm marker CXCR4 was highest in Stage 3 iHIOs and transwell cells, which went through a definitive endoderm intermediate at Stage 1. Similarly, expression of the mesenchymal marker Vimentin was also most highly upregulated in iPSC-derived intestinal cells, relative to Caco-2. This result is consistent with previously reported increases in expression of Vimentin and another mesenchymal marker, Forkhead Box F1 (Mahlapuu et al., 1998), in intestinal cells derived from human embryonic stem cells, any may indicate the development of intestinal subepithelial myofibroblasts (Spence et al., 2010).

The intestinal stem cell marker LGR-5 (Barker et al., 2007) was highly upregulated in iPSC-derived intestinal cells relative to Caco-2, which may reflect more active intestinal cell proliferation within these cells, particularly in iHIOs. Expression of Paired Box Gene 6 (PAX6), with known roles in brain development (Mastick et al., 1997), was also highly expressed in iHIOs cells, with less expression in Stage 3 iPSC-derived transwell cells or the other intestinal sources tested. This is consistent with the high Chromogranin A expression in the development of a more mature enteroendocrine system within these cells, as PAX6 also has a known role in the development of Glucagon-like peptide (GLP)-1 and GLP-2 secreting cells (Fujita et al., 2008; Ye and Kaestner, 2009).

While intestinal marker expression in primary of iPSC-derived cells was very encouraging, it was unclear how functional these cells are compared to other established in vitro intestinal models, such as Caco-2 or primary tissue. For example, in pluripotent stem-cell derived models of liver or pancreas, progenitor cells must be engrafted into whole animals to produce mature, fullyfunctional cell types (Liu et al., 2011; Rezania et al., 2011). We realized that adaptation of directed intestinal organogenesis to 2D transwell culture would allow a more direct comparison of iPSC-derived intestinal cell barrier and transport functions to previously characterized intestinal epithelial cell models in vitro. Well-studied intestinal transport mechanisms within Caco-2 and primary human intestinal tissue include efflux of small molecules by membrane associated Pgp transporters, (Murakami and Takano, 2008), and intestinal receptor-mediated antibody transcytosis by FcRn (Dickinson et al., 1999; Claypool et al., 2004). These mechanisms were used as an initial assessment of functional quality of primary hInEpCs and iPSC-derived intestinal cells.

Primary hInEpCs A and B showed cyclosporine A-dependent efflux of Digoxin, but not Atenolol, indicative of the presence of Pgp transport activity in these cells. In addition to primary hInEpCs (Donor A), Stage 3 iPSC-derived transwell cells showed FcRn expression and FcRn-dependent mAb binding using high or low FcRn-binding IgG variants; however, unlike primary hInEpCs, iPSC-derived IgG binding did not occur to as large an extent as Caco-2. It should also be noted that in all FcRnbinding experiments, the cell binding signal, while well above background noise, was relatively low for this ELISA-based assay. Furthermore, since FcRn is expressed in neonatal and adult intestinal epithelial cells in primates (Israel et al., 1997; Dickinson et al., 1999) and marker expression during differentiation of iHIOs closely resembles that of embryonic intestinal development (Spence et al., 2010), it is still unclear whether the phenotype of these cells more closely resembles embryonic or adult intestinal tissue.

In summary, our studies indicate that iPSC-derived intestinal cells and newly commercially available primary hInEpCs may provide an alternative source of physiologically relevant hInEpCs. Future studies will be needed to further evaluate the function of primary hInEpCs and iPSC-derived 
intestinal cells. Additionally, as these cells are still limited by their viability (hInEpCs) and extensive differentiation time (iPSCderived cells), large-scale studies will require additional development of methods for scale up or long-term maintenance of these transwell cultures, such as non-oncogenic immortalization strategies.

\section{REFERENCES}

Aldhous, M. C., Shmakov, A. N., Bode, J., and Ghosh, S. (2001). Characterization of conditions for the primary culture of human small intestinal epithelial cells. Clin. Exp. Immunol. 125, 32-40. doi: 10.1046/ j.1365-2249.2001.01522.x

Balimane, P. V., and Chong, S. (2005). Cell culture-based models for intestinal permeability: a critique. Drug Discov. Today 10, 335-43. doi: 10.1016/S1359-6446(04)03354-9

Balimane, P. V., Han, Y. H., and Chong, S. (2006). Current industrial practices of assessing permeability and P-glycoprotein interaction. AAPS J. 8, E1-E13. doi: 10.1208/ aapsj080101

Barker, N., Van Es, J. H., Kuipers, J., Kujala, P., Van Den Born, M., Cozijnsen, M., et al. (2007). Identification of stem cells in small intestine and colon by marker gene Lgr5. Nature 449, 1003-1007. doi: 10.1038/nature06196

Bell, S. M., Zhang, L., Xu, Y., Besnard, V., Wert, S. E., Shroyer, N., et al. (2013). Kruppel-like factor 5 controls villus formation and initiation of cytodifferentiation in the embryonic intestinal epithelium. Dev. Biol. 375, 128-139. doi: 10.1016/j.ydbio. 2012.12.010

Bosch, F. X., Leube, R. E., Achtstatter, T., Moll, R., and Franke, W. W. (1988). Expression of simple epithelial type cytokeratins in stratified epithelia as detected by immunolocalization and hybridization in situ. J. Cell Biol. 106, 1635-1648. doi: 10.1083/jcb.106.5.1635

Claypool, S. M., Dickinson, B. L., Wagner, J. S., Johansen, F. E., Venu, N., Borawski, J. A., et al. (2004). Bidirectional transepithelial IgG transport by a strongly polarized basolateral membrane Fcgamma-receptor. Mol. Biol. Cell 15, 1746-1759. doi: 10.1091/mbc. E03-11-0832

Dickinson, B. L., Badizadegan, K., Wu, Z., Ahouse, J. C., Zhu, X., Simister, N. E., et al. (1999). Bidirectional FcRn-dependent IgG transport in a polarized human intestinal epithelial cell line. J. Clin. Invest. 104, 903-911. doi: 10.1172/JCI6968

Friederich, E., Vancompernolle, K., Louvard, D., and Vandekerckhove, J.
(1999). Villin function in the organization of the actin cytoskeleton. Correlation of in vivo effects to its biochemical activities in vitro. J. Biol. Chem. 274, 26751-26760. doi: 10.1074/jbc.274.38.26751

Fujita, Y., Chui, J. W., King, D. S., Zhang, T., Seufert, J., Pownall, S., et al. (2008). Pax6 and Pdx1 are required for production of glucose-dependent insulinotropic polypeptide in proglucagonexpressing L cells. Am. J. Physiol. Endocrinol. Metab. 295, E648-E657. doi: 10.1152/ajpendo.90440.2008

Gao, N., White, P., and Kaestner, K. H. (2009). Establishment of intestinal identity and epithelialmesenchymal signaling by $\mathrm{Cdx} 2$. Dev. Cell 16, 588-99. doi: 10.1016/ j.devcel.2009.02.010

Gitter, A. H., Bendfeldt, K. Schulzke, J. D., and Fromm, M. (2000). Trans/paracellular, surface/crypt, and epithelial/subepithelial resistances of mammalian colonic epithelia Pflugers Arch. 439, 477-482. doi: 10.1007/s004240050966

Gitter, A. H., Fromm, M., and Schulzke, J. D. (1998). Impedance analysis for the determination of epithelial and subepithelial resistance in intestinal tissues. J. Biochem. Biophys. Methods 37, 35-46. doi: 10.1016/S0165-022X (98)00016-5

Gum, J. R. Jr., Hicks, J. W., Gillespie, A. M., Carlson, E. J., Komuves, L., Karnik, S., et al. (1999). Goblet cellspecific expression mediated by the MUC2 mucin gene promoter in the intestine of transgenic mice. Am. J. Physiol. 276, G666-G676.

Israel, E. J., Taylor, S., Wu, Z., Mizoguchi, E., Blumberg, R. S. Bhan, A., et al. (1997). Expression of the neonatal $\mathrm{Fc}$ receptor, $\mathrm{FcRn}$, on human intestinal epithelial cells. Immunology 92, 69-74. doi: $\quad 10.1046 / j .1365-2567.1997$. 00326.x

Lahar, N., Lei, N. Y., Wang, J., Jabaji, Z., Tung, S. C., Joshi, V., et al. (2011). Intestinal subepithelial myofibroblasts support in vitro and in vivo growth of human small intestinal epithelium. PLoS ONE 6:e26898. doi: 10.1371/journal.pone.0026898

Le Ferrec, E., Chesne, C., Artusson, P., Brayden, D., Fabre, G., Gires,

\section{ACKNOWLEDGMENTS}

We thank Nathan Majewski, Jason Ekert, and Kimberly Reese for help with iPSC maintenance culture. We also acknowledge the outstanding quality of immunohistochemistry performed by Steve Bernstein and Marko Sldanov, Qualtek Molecular Labs, Goleta, CA.

P., et al. (2001). In vitro models of the intestinal barrier. The report and recommendations of ECVAM workshop 46. European centre for the validation of alternative methods. Altern. Lab. Anim. 29, 649-668.

Leonard, M., Creed, E., Brayden, D., and Baird, A. W. (2000). Evaluation of the Caco-2 monolayer as a model epithelium for iontophoretic transport. Pharm. Res. 17, 1181-8. doi: 10.1023/A:1026454427621

Liu, H., Kim, Y., Sharkis, S. Marchionni, L., and Jang, Y. Y. (2011). In vivo liver regeneration potential of human induced pluripotent stem cells from diverse origins. Sci. Transl. Med. 3, 82ra39. doi: 10.1126/scitranslmed. 3002376

Mahlapuu, M., Pelto-Huikko, M. Aitola, M., Enerback, S., and Carlsson, P. (1998). FREAC-1 contains a cell-type-specific transcriptional activation domain and is expressed in epithelialmesenchymal interfaces. Dev. Biol. 202, 183-195. doi: 10.1006/dbio. 1998.9010

Mastick, G. S., Davis, N. M., Andrew, G. L., and Easter, S. S. Jr. (1997). Pax6 functions in boundary formation and axon guidance in the embryonic mouse forebrain. Development 124, 1985-1997.

McCracken, K. W., Howell, J. C., Wells, J. M., and Spence, J. R. (2011). Generating human intestinal tissue from pluripotent stem cells in vitro. Nat. Protoc. 6, 1920-1928. doi: 10.1038/nprot.2011.410

Miller, C. P., McGehee, R. E. Jr., and Habener, J. F. (1994). IDX-1: a new homeodomain transcription factor expressed in rat pancreatic islets and duodenum that transactivates the somatostatin gene. EMBO J. 13 , 1145-1156.

Murakami, T., and Takano, M. (2008). Intestinal efflux transporters and drug absorption. Expert Opin. Drug Metab. Toxicol. 4, 923-939. doi: 10.1517/17425255.4.7.923

Nichols, J., Zevnik, B., Anastassiadis, K., Niwa, H., Klewe-Nebenius, D., Chambers, I., et al. (1998). Formation of pluripotent stem cells in the mammalian embryo depends on the POU transcription factor Oct4. Cell 95, 379-391. doi: 10.1016/S0092-8674(00)81769-9

O'Connor, D. T., Burton, D., and Deftos, L. J. (1983). Chromogranin A: immunohistology reveals its universal occurrence in normal polypeptide hormone producing endocrine glands. Life Sci. 33, 1657-1663. doi: 10.1016/0024-3205 (83)90721-X

Ootani, A., Li, X., Sangiorgi, E., Ho, Q. T., Ueno, H., Toda, S., et al. (2009). Sustained in vitro intestinal epithelial culture within a Wntdependent stem cell niche. Nat. Med. 15, 701-706. doi: 10.1038/nm. 1951

Peeters, T., and Vantrappen, G. (1975) The Paneth cell: a source of intestinal lysozyme. Gut 16, 553-558. doi: 10.1136/gut.16.7.553

Perreault, N., and Beaulieu, J. F. (1998). Primary cultures of fully differentiated and pure human intestinal epithelial cells. Exp. Cell Res. 245, 34-42. doi: 10.1006/excr.1998.4221

Poirier, K., Saillour, Y., Bahi-Buisson, N., Jaglin, X. H., Fallet-Bianco, C., Nabbout, R., et al. (2010). Mutations in the neuronal sstubulin subunit TUBB3 result in malformation of cortical development and neuronal migration defects. Hum. Mol. Genet. 19, 4462-4473. doi: 10.1093/hmg/ ddq377

Rezania, A., Riedel, M. J., Wideman, R. D., Karanu, F., Ao, Z., Warnock, G. L., et al. (2011). Production of functional glucagon-secreting alpha-cells from human embryonic stem cells. Diabetes 60, 239-247. doi: $10.2337 / \mathrm{db} 10-0573$

Sato, T., Vries, R. G., Snippert, H. J., Van De Wetering, M., Barker, N., Stange, D. E., et al. (2009). Single Lgr5 stem cells build crypt-villus structures in vitro without a mesenchymal niche. Nature 459, 262-265. doi: 10.1038/nature07935

Spence, J. R., Mayhew, C. N., Rankin, S. A., Kuhar, M. F., Vallance, J. E., Tolle, K., et al. (2010). Directed differentiation of human pluripotent stem cells into intestinal tissue in vitro. Nature 470, 105-109. doi: 10.1038/ nature09691

Takahashi, K., Tanabe, K., Ohnuki, M. Narita, M., Ichisaka, T., Tomoda, K., et al. (2007). Induction of 
pluripotent stem cells from adult human fibroblasts by defined factors. Cell 131, 861-872. doi: 10.1016/j.cell.2007.11.019

Townsend, P. J., Farza, H., Macgeoch, C., Spurr, N. K., Wade, R., Gahlmann, R., et al. (1994). Human cardiac troponin $\mathrm{T}$ : identification of fetal isoforms and assignment of the TNNT2 locus to chromosome 1q. Genomics 21, 311-316. doi: 10.1006/geno.1994.1271

van der Flier, L. G., Van Gijn, M. E. Hatzis, P., Kujala, P., Haegebarth, A., Stange, D. E., et al. (2009). Transcription factor achaete scutelike 2 controls intestinal stem cell fate. Cell 136, 903-912. doi: 10.1016/j.cell.2009.01.031

Watanabe, T., Kokubu, N., Charnick, S. B., Naito, M., Tsuruo, T., and Cohen, D. (1997). Interaction of cyclosporin derivatives with the ATPase activity of human P-glycoprotein. Br. J. Pharmacol. 122, 241-248. doi: 10.1038/sj.bjp. 0701377

Yakubov, E., Rechavi, G., Rozenblatt, S., and Givol, D. (2010). Reprogramming of human fibroblasts to pluripotent stem cells using mRNA of four transcription factors. Biochem. Biophys. Res. Commun 394, 189-193. doi: 10.1016/j.bbrc. 2010.02.150

Ye, D. Z., and Kaestner, K. H. (2009). Foxal and Foxa2 control the differentiation of goblet and enteroendocrine L- and D-cells in mice. Gastroenterology 137, 2052-2062. doi: 10.1053/j.gastro. 2009.08.059

Yu, H., and Sinko, P. J. (1997). Influence of the microporous substratum and hydrodynamics on resistances to drug transport in cell culture systems: calculation of intrinsic transport parameters. J. Pharm. Sci. 86, 1448-1457. doi: 10.1021/ js960524q

Zbar, A. P., Simopoulos, C., and Karayiannakis, A. J. (2004). Cadherins: an integral role in inflammatory bowel disease and mucosal restitution. J. Gastroenterol. 39, 413-421. doi: 10.1007/s00535004-1335-8

Conflict of Interest Statement: All investigators are employees of Johnson \& Johnson and have no other declarations of interest to disclose.

Received: 26 April 2013; paper pending published: 17 May 2013; accepted:
03 June 2013; published online: 08 July 2013.

Citation: Kauffman AL, Gyurdieva AV, Mabus JR, Ferguson C, Yan $Z$ and Hornby PJ (2013) Alternative functional in vitro models of human intestinal epithelia. Front. Pharmacol. 4:79. doi: 10.3389/fphar.2013.00079

This article was submitted to Frontiers in Gastrointestinal Pharmacology, a specialty of Frontiers in Pharmacology.

Copyright (c) 2013 Kauffman, Gyurdieva, Mabus, Ferguson, Yan and Hornby. This is an open-access article distributed under the terms of the Creative Commons Attribution License, which permits use, distribution and reproduction in other forums, provided the original authors and source are credited and subject to any copyright notices concerning any third-party graphics etc. 


\section{APPENDIX \\ RESULTS}

In addition to intestinal cell-specfic markers (Figure 4), other control markers were assessed by RT-PCR to evaluate sample quality of iPSC-derived cells, Caco-2, and primary intestinal cells (Figure A2). iPSC-derived cells and primary intestinal cells showed very low expression of pluripotent cell transcription factor POU domain, class 5 (OCT4) (Nichols et al., 1998), and about 2000-fold lower than undifferentiated cells (Figure A2A). To determine how successfully iPSC-derived intestinal cells differentiated away from a definitive endoderm cell type after Stage 1, expression of CXCR4 was analyzed. As somewhat expected, expression of this definitive endoderm marker was highest in iPSC-derived cells, which went through a definitive endoderm intermediate at Stage 1, with expression levels ranging about 150-fold higher relative to Caco-2 (Figure A2B). Expression of the pancreatic foregut marker PDX1 (Miller et al., 1994) was found to be 10-15-fold higher in iPSC-derived cells and primary hInEpCs relative to Caco-2 (Figure A2C). Similarly, expression of the mesenchymal marker Vimentin was also highly upregulated in primary hInEpCs and iPSC-derived intestinal cells, relative to Caco-2 (Figure A2D).

Neuronal marker Tubulin, beta 3 (TUBB3) (Poirier et al., 2010), expression was higher in iPSC-derived cells and primary hInEpCs relative to Caco-2 (Figure A2E). Expression of cardiac cell marker Troponin T, type 2 (TNNT2) (Townsend et al., 1994) was the comparable across intestinal cell types (Figure A2F). Expression of Paired Box Gene 6 (PAX6), with known roles in brain development (Mastick et al., 1997), was highly expressed only Stage 3 iHIOs (Figure A2G).

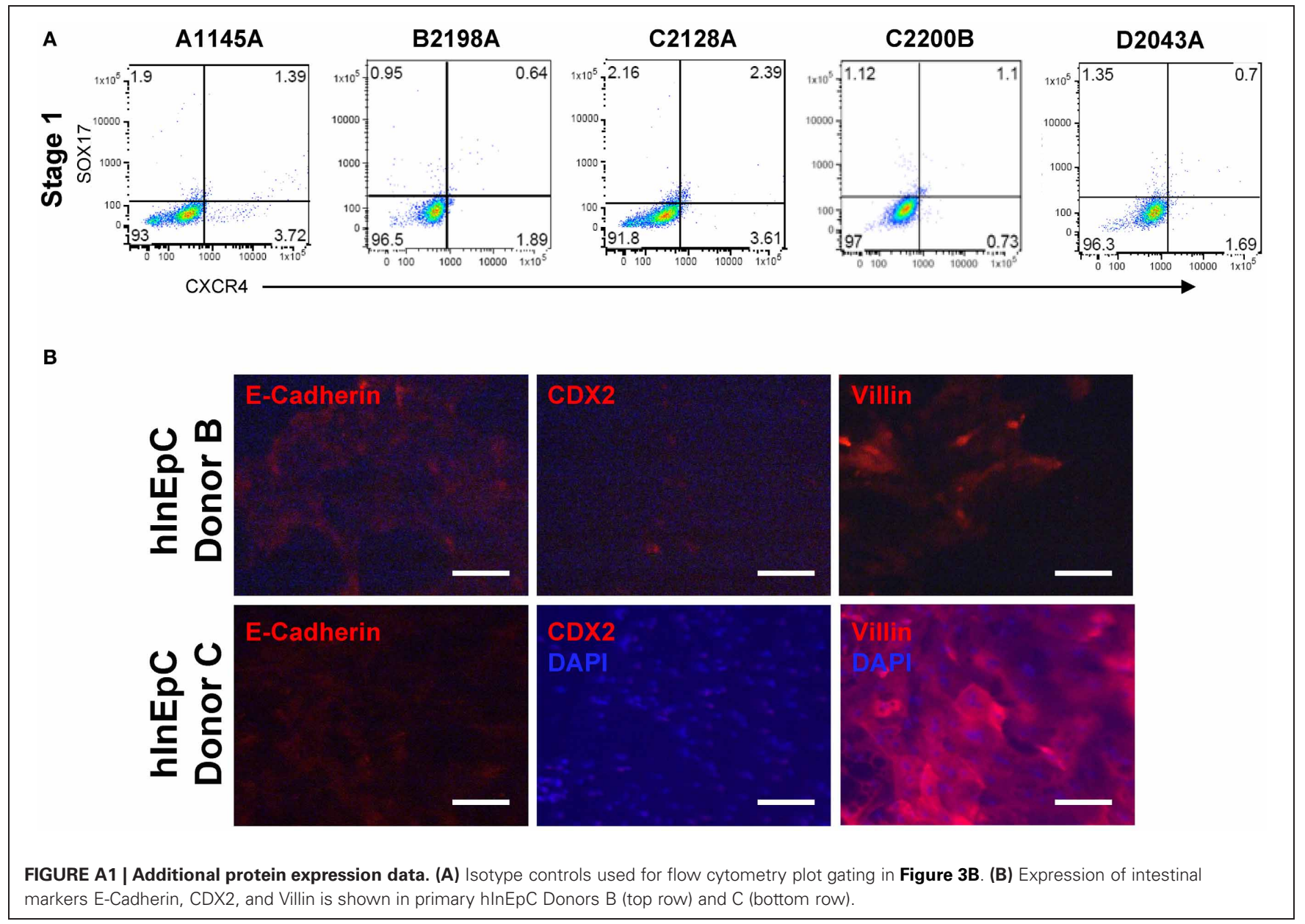




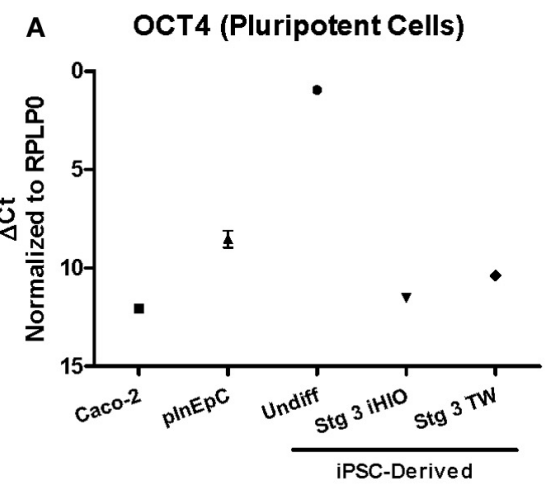

C

PDX1 (Foregut/Pancreas)

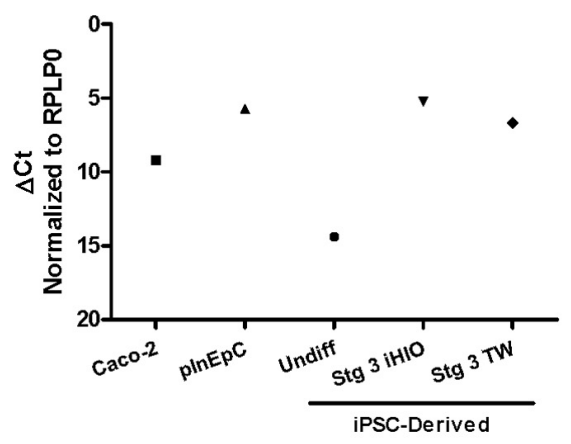

E

TUBB3 (Late Neuronal Development)

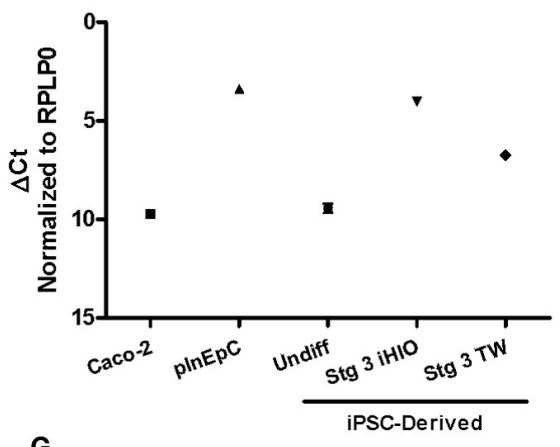

G

PAX6 (Early Neuronal Development)

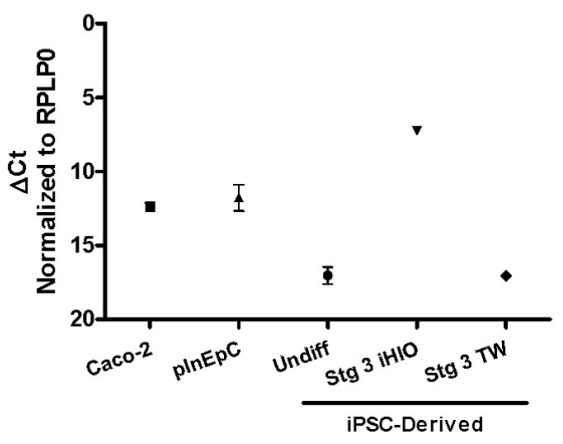

FIGURE A2 | Validation of intestinal cell RT-PCR analysis through a panel of control/non-intestinal markers. Expression of intestinal markers was evaluated by RT-PCR in Caco-2, primary hlnEpCs

(average of Donors $\mathrm{A}-\mathrm{C}$ ), and iPSC-derived undifferentiated (Undiff),
$\mathbf{B}$

CXCR4 (Definitive Endoderm)

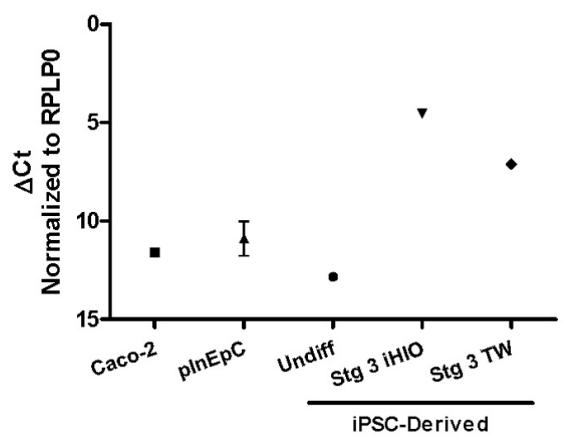

D

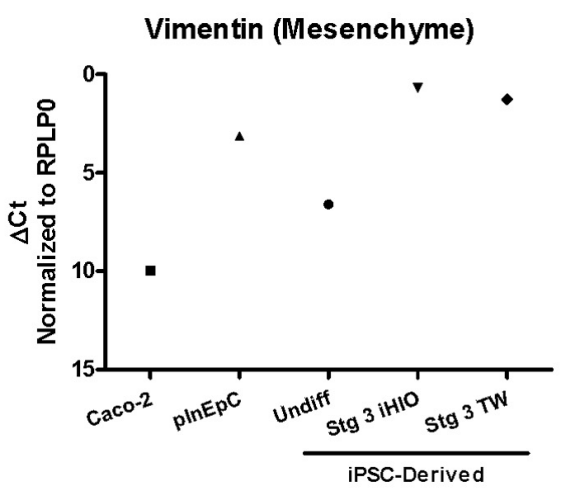

$\mathbf{F}$

TNNT 2 (Cardiac Cells)

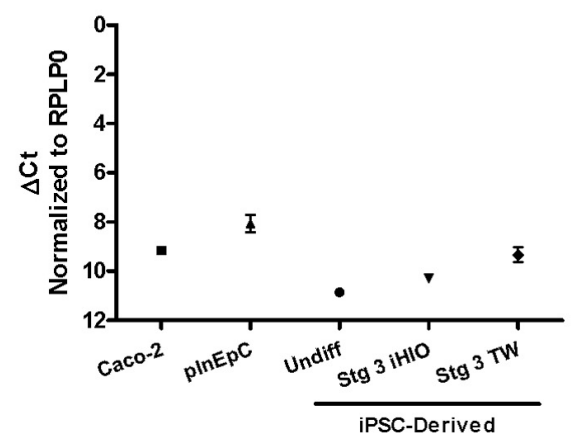

Stage 3 induced human intestinal organoids (Stg 3 iHIOs), or Stage 3 transwell intestinal cells (Stg 3 TW). Using the DCt method, raw $C t$ values were normalized to housekeeping gene RPLPO. $N=3$; Error bars represent SEM 


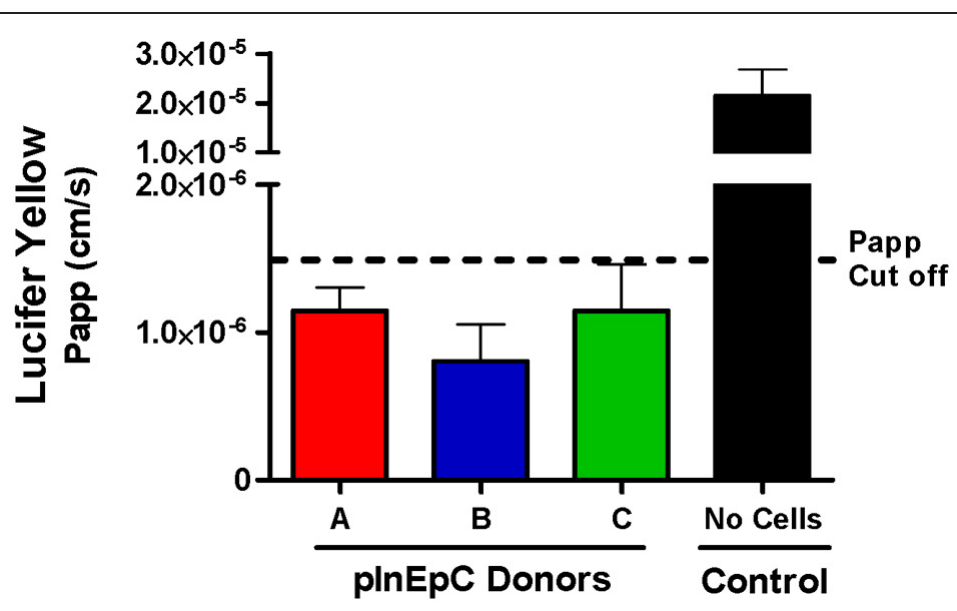

FIGURE A3 | All primary hlnEpC donors used within the P-glycoprotein $\mathbf{1 . 5} \times \mathbf{1 0}^{\mathbf{- 6}} \mathbf{~} \mathbf{m} / \mathbf{s}$. Only transwells that met this cutoff were used to transport assay showed an average lucifer yellow apparent permeability (Papp) below accepted cutoff for intact monolayers calculate Papp ratios for Digoxin and Atenolol (Figure 6). $N=3$; Error bars represent SEM.

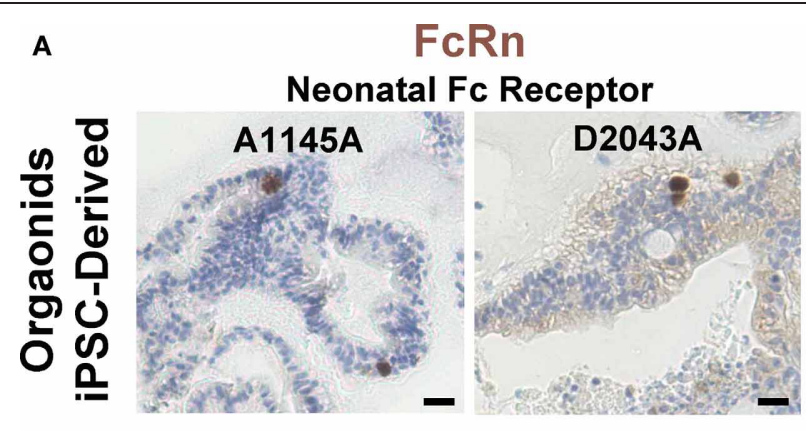

B

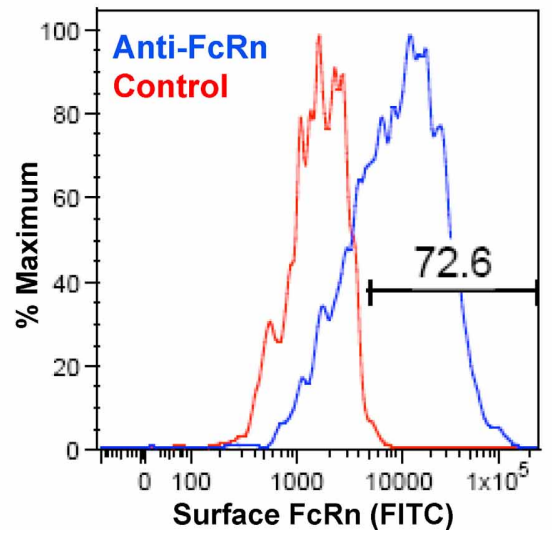

FIGURE A4 | Intestinal epithelial cell expression of FcRn. (A)

Immunohistochemical analysis demonstrates iPSC-derived Stage 3 intestinal organoid expression and punctate localization of neonatal Fc Receptor
Primary hInEpC Donor A

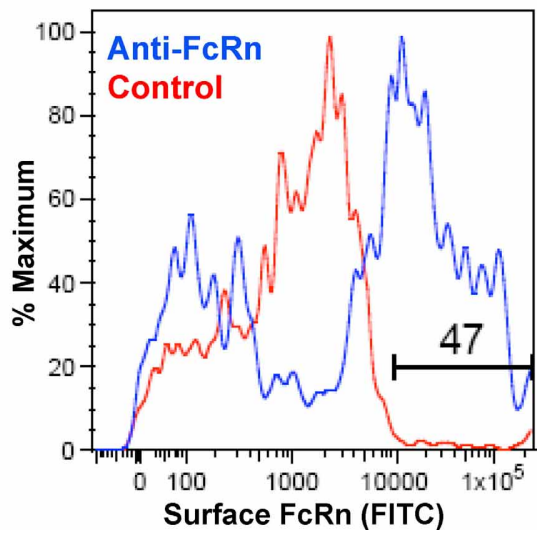

expression in iPSC-derived organoids. Scale bar, $100 \mu \mathrm{m}$. (B) Flow cytometry analysis shows surface FcRn expression on Caco-2 (left) and primary hlnEpCs (right, Donor A shown). 


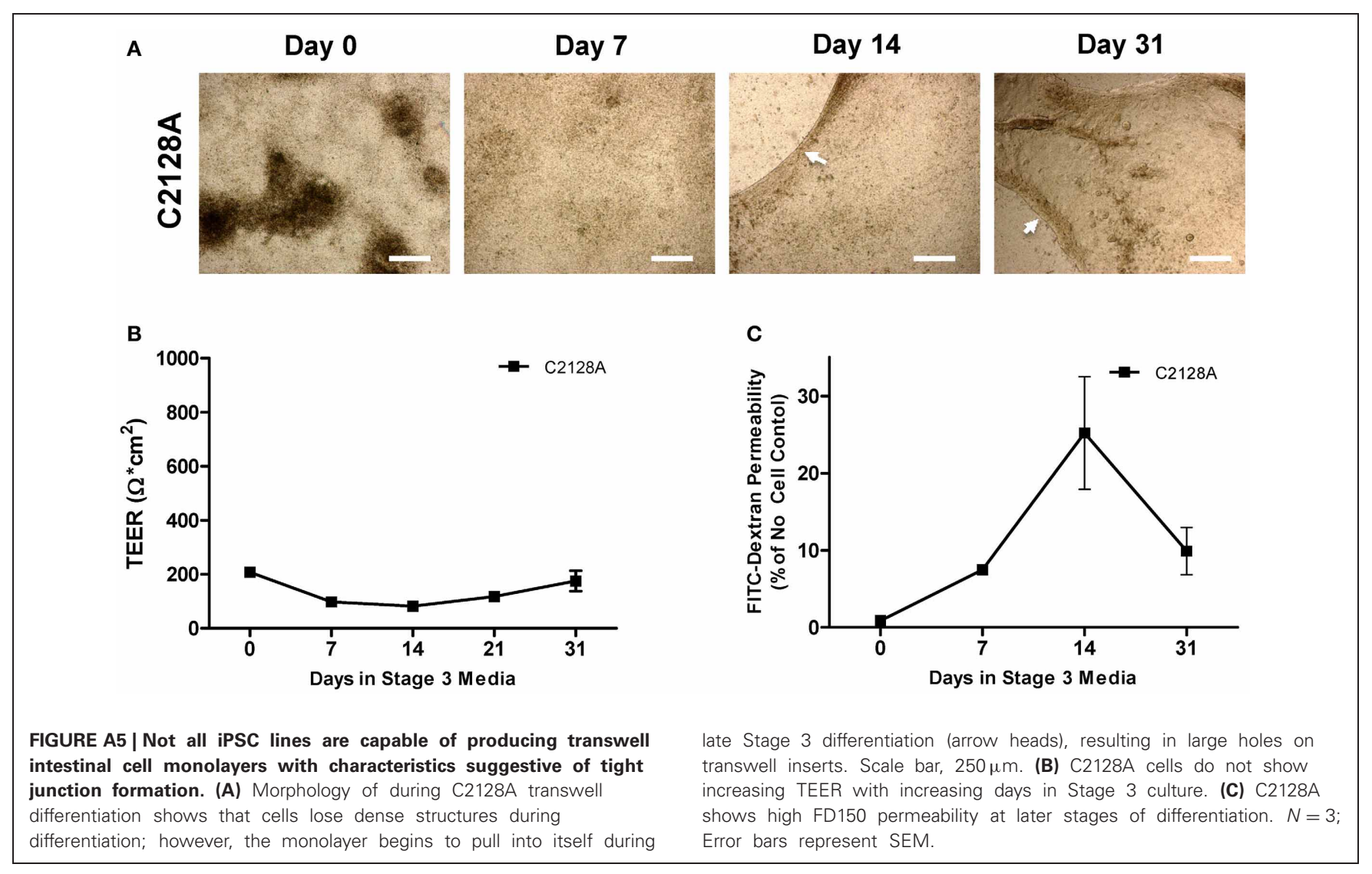




\section{REFERENCES}

Mastick, G. S., Davis, N. M., Andrew, G. L., and Easter, S. S. Jr. (1997). Pax6 functions in boundary formation and axon guidance in the embryonic mouse forebrain. Development 124, 1985-1997.

Miller, C. P., McGehee, R. E. Jr., and Habener, J. F. (1994). IDX-1: a new homeodomain transcription factor expressed in rat pancreatic islets and duodenum that transactivates the somatostatin gene. $E M B O J .13$, 1145-1156.

Nichols, J., Zevnik, B., Anastassiadis, K., Niwa, H., Klewe-Nebenius, D., Chambers, I., et al. (1998). Formation of pluripotent stem cells in the mammalian embryo depends on the POU transcription factor Oct4. Cell 95, 379-391. doi: 10.1016/S0092-8674(00)81769-9

Poirier, K., Saillour, Y., Bahi-Buisson,

N., Jaglin, X. H., Fallet-Bianco,

C., Nabbout, R., et al. (2010).

Mutations in the neuronal sstubulin subunit TUBB3 result in malformation of cortical development and neuronal migration defects. Hum. Mol. Genet. 19,
4462-4473. doi: $\quad 10.1093 / \mathrm{hmg} /$ ddq377

Townsend, P. J., Farza, H., Macgeoch, C., Spurr, N. K., Wade, R., Gahlmann, R., et al. (1994). Human cardiac troponin $\mathrm{T}$ : identification of fetal isoforms and assignment of the TNNT2 locus to chromosome 1q. Genomics 21, 311-316. doi: 10.1006/geno.1994.1271 